\title{
Oncocytic Renal Cell Carcinoma with Tubulopapillary Growth Having a Fat Component
}

\author{
$\mathrm{Na}$ Rae Kim $\cdot$ Hyun Yee Cho \\ Department of Pathology, Gachon University Gil \\ Medical Center, Incheon, Korea \\ Received: April 24, 2015 \\ Revised: June 3, 2015 \\ Accepted: July 1, 2015

\section{Corresponding Author} \\ Hyun Yee Cho, MD \\ Department of Pathology, Gachon University Gil \\ Medical Center, 21 Namdong-daero 774 beon-gil, \\ Namdong-gu, Incheon 21565, Korea \\ Tel: +82-32-460-3865 \\ Fax: +82-32-460-2394 \\ E-mail: hicho@gilhospital.com
}

\begin{abstract}
We report a rare case of oncocytic renal cell carcinoma $(\mathrm{RCC})$ with tubulopapillary growth in the background of tuberculous end-stage kidney disease. Histology of the renal mass consisted of oncocytic cells forming solid, thin tubules and rare papillae. The tumor had abundant eosinophilic oncocytic cells containing occasional cytoplasmic Mallory body-like hyaline globules and a tiny focus of clear cells with intervening mature fat. Both the oncocytic cells and clear cells were immunoreactive for a-methylacyl-CoA racemase, vimentin, pancytokeratin, and CD10, and negative for transcription factor E3, CD15, human melanoma black 45, and c-kit. Mallory body-like hyaline globules were positive for CAM 5.2 and periodic acid-Schiff with or without diastase. Ultrastructurally, the tumor cells had abundant cytoplasmic mitochondria. The present case is a rare case of oncocytic RCC with tubulopapillary growth pattern. The case is unique in that the tumor was mixed with fat component, which is not common in RCC and thus can lead to misdiagnosis.
\end{abstract}

Key Words: Oxyphil cells; Carcinoma, renal cell; Adipocytes; Mycobacterium tuberculosis
Recent advances in cytogenetics have altered the traditional classification of renal cell carcinoma (RCC). According to the latest World Health Organization classification, renal cell tumors are classified based on their morphological, immunohistochemical, and genetic features. ${ }^{1}$ The following subgroups can be used to classify these tumors: clear cell RCC (VHL and others on chromosome 3), papillary RCC (MET), chromophobe RCC (TP53), and collecting duct RCC. ${ }^{2}$ Among them, papillary RCC can display extensive areas of solid and non-papillary architecture and extensive areas of oncocytic cytoplasm. As a rare morphological variant of papillary RCC, oncocytic RCC has been described as having papillotubular growth, as oncocytic papillary RCC with an inverted nuclear pattern, or as oncocytic papillary RCC with solid architecture. ${ }^{3-6}$ This new variant is composed of tumor cells with morphological, immunohistochemical, and ultrastructural characteristics of oncocytes, and frequently presents as high nuclear grade 3, type 2 papillary RCC with rare metastases, similar to type 1 papillary RCC. The present case of an oncocytic variant of RCC coexisted with renal tuberculosis.

Here, we report a rare case of oncocytic RCC with a tubulopapillary growth pattern and extracellular mature fat component in a tuberculous end-stage kidney and discuss its complex differential diagnosis.

\section{CASE REPORT}

A 52-year-old male had been treated for chronic renal failure and had been on continuous ambulatory peritoneal dialysis for 9 years. He visited our hospital due to a recent onset of vague left flank pain. Abdominopelvic computed tomography (CT) revealed a round mass in the lower pole of the left kidney and marked hydronephroureter with cortical thinning of bilateral kidneys (Fig. 1). Chest CT showed segmental consolidation with surrounding ground glass opacity in the right upper lobe, suggesting pulmonary tuberculosis. Bilateral nephrectomy was then performed.

Grossly, a well-circumscribed, round, solid mass measuring $2.5 \times 2.0 \times 1.5 \mathrm{~cm}$ was found at the lower pole of the left kidney. The cut surface of the mass was yellow-tan colored. Both kidneys showed severe hydronephrosis and pyelonephritic changes. The dilated pelvocalyces were covered with yellow-tan necrotic material. Microscopically, most of the mass was composed of solid and thin tubules of eosinophilic oncocytic cells mixed with mature fat (Fig. 2A, B). The polygonal-shaped tumor cells had abundant eosinophilic granular cytoplasm and round nuclei with prominent nucleoli (Fig. 2C). Elongated tubular architectures were predominant with occasional detachment from the lumens 


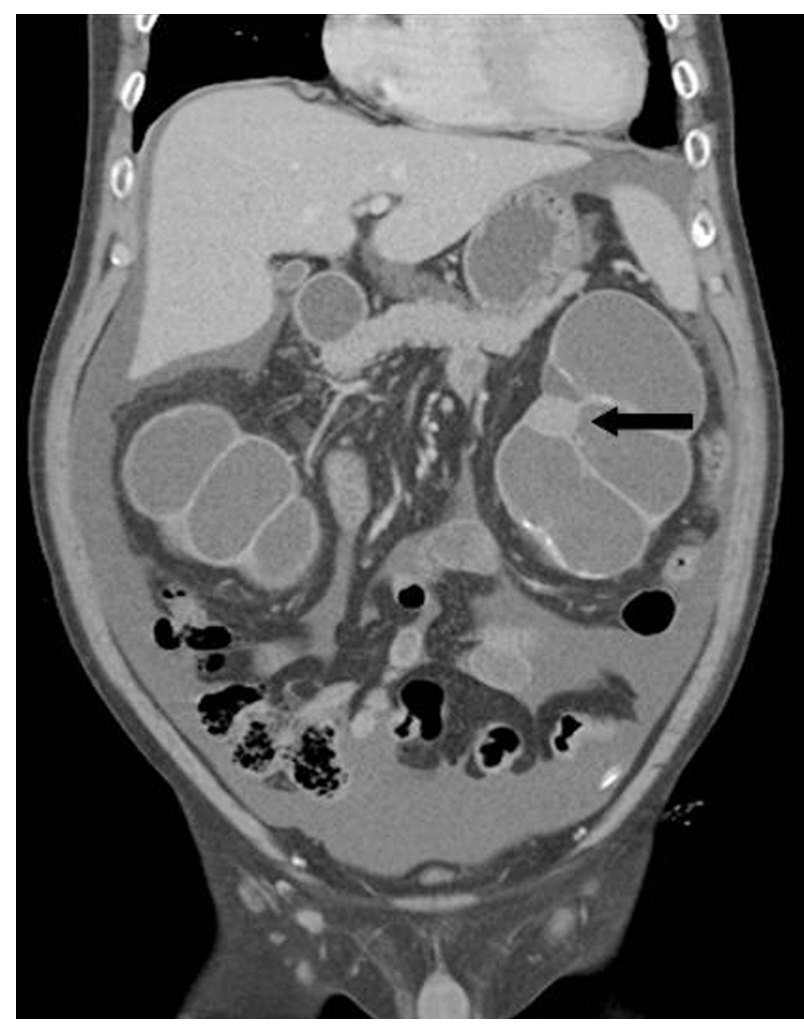

Fig. 1. Coronal view of abdominopelvic computed tomography reveals severe hydronephrosis of bilateral kidneys. Note a round lesion in the left kidney (arrow).

(Fig. 2D). The tumor demonstrated only focal large papillary fronds that contained delicate fibrovascular cores (Fig. 2E, left) and aggregates of clear cells (Fig. 2E, right). Occasionally, the oncocytic cells had Mallory body-like eosinophilic cytoplasmic hyaline globules (Fig. 2F, left). Mitotic figures were counted up to 2 per 10 high power fields. The surrounding parenchyma showed a caseous necrotic cystic wall. Immunohistochemically, both the oncocytic and clear cells were diffusely strongly positive for vimentin (prediluted, V9, Dako, Glostrup, Denmark), pancytokeratin (prediluted, AE1/AE3, Dako), and $\alpha$-methylacylCoA racemase (AMACR; 1:50, EPUM1, Novocastra, New Castle upon Tyne, UK). Some of the oncocytic cells were weakly positive for CD10 (1:100, 56C6, Dako). The tumor cells were negative for epithelial membrane antigen (prediluted, E29, Dako), cytokeratin 7 (CK7; 1:100, OV-TL, Dako), MOC31 (MOC31; 1:70, Novocastra), E-cadherin (1:50, 36B5, Dako), carcinoembryonic antigen (prediluted, Dako), progesterone receptor (1:50, PgR636, Dako), CD15 (1:50, C3D-1, Dako), human melanoma black 45 (HMB-45; prediluted, Dako), transcription factor E3 (1:50, MRQ-37, Cell Marque Corporation, Rocklin, CA, USA), and c-kit (1:30, T595, Novocastra). These cells were also negative for Hale's colloidal iron and periodic acid-Schiff (PAS) staining. Mallory body-like eosinophilic hyaline globules were stained with PAS and diastase-resistant (Fig. 2F, right), and they were slightly positive for CAM 5.2 (prediluted, BD Bioscience, San Diego, CA, USA) and negative for $\alpha$-fetoprotein (prediluted, Dako). The tumor was diagnosed as oncocytic papillary RCC of Fuhrman's nuclear grade 3. Surrounding parenchyma as well as renal pelvis and ureter showed chronic granulomatous inflammation with caseation necrosis. The oncocytic areas of the paraffin block were taken for ultrastructural examination. Electron microscopy revealed that the ovoid-shaped tumor cells had abundant cytoplasm and round nuclei with a euchromatic pattern. They had abundant mitochondria and some rough endoplasmic reticula (Fig. 3).

The mass was diagnosed as oncocytic RCC with a tubulopapillary growth pattern. During 9 years of postoperative follow up, the patient showed no recurrence or metastasis.

Institutional Review Board (IRB) of Gachon University Gil Medical Center (IRB No. GCIRB2015-192) approval was obtained for this case report.

\section{DISCUSSION}

Under light microscopy, the present renal tumor showed the characteristic pathologic findings of oncocytic tumor composed of mainly solid architecture with thin tubules, i.e., short papillae and intermixed fat. There are several oncocytic renal cell neoplasms with these histopathological features, which poses a diagnostic problem in differentiation between RCC with oncocytic features, oncocytoma-like epithelioid angiomyolipoma (AML), and renal oncocytoma. ${ }^{1}$ Renal oncocytoma shows typical gross features of a solitary, well-circumscribed, slightly lobulated, solid appearance with a mahogany brown or dark red cut surface with a frequent central scar. Microscopically, the small, round, uniform tumor cells of oncocytoma have abundant, faintly eosinophilic granular cytoplasm, and the cells are arranged in nests and tubulocystic, solid, or trabecular patterns with a myxomatous or hyalinized stroma. Immunohistochemistry can be helpful for arriving at the correct diagnosis because the distinct immunopositivity for c-kit and the immunonegativity for $\mathrm{CK} 7$ and vimentin are characteristic of renal oncocytoma. ${ }^{1}$ Clear cell RCC has diverse architecture of solid, alveolar, and acinar patterns, but prominent papillary architecture is not common. An extensive papillary architecture is one of the characteristic features of papillary RCC. A focal tubular growth pattern with small papillae may be also seen in papillary RCC, but extensive 

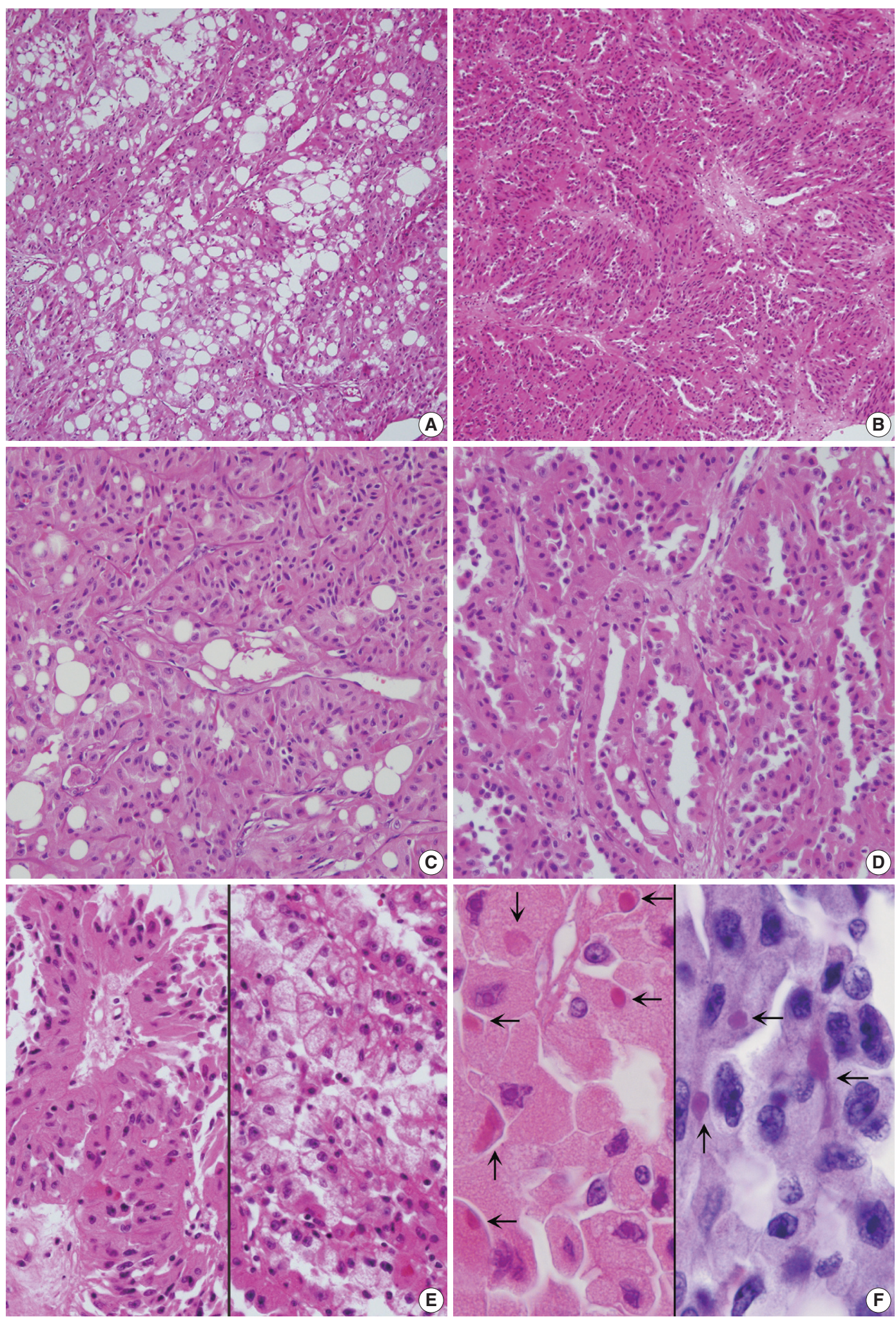

Fig. 2. (A) Tumor cells with abundant eosinophilic cytoplasm are arranged in solid papillae and tubules, intermingled with mature fat component. (B) The tumor is composed of a compact arrangement of small thin tubules. (C) At the solid area, lobules separated by thin capillaries consist of eosinophilic and granular tumor cells. Note fat vacuoles. (D) Eosinophilic tumor cells are lining the tubules with focal surface decapitation. (E) Oncocytic cells occasionally form papillary architecture (left). The tubules of clear tumor cells have the appearance of clear-type renal cell carcinoma (right). (F) The oncocytic cells have eosinophilic cytoplasmic globules (arrows, left) that are stained with periodic acid-Schiff (right). 


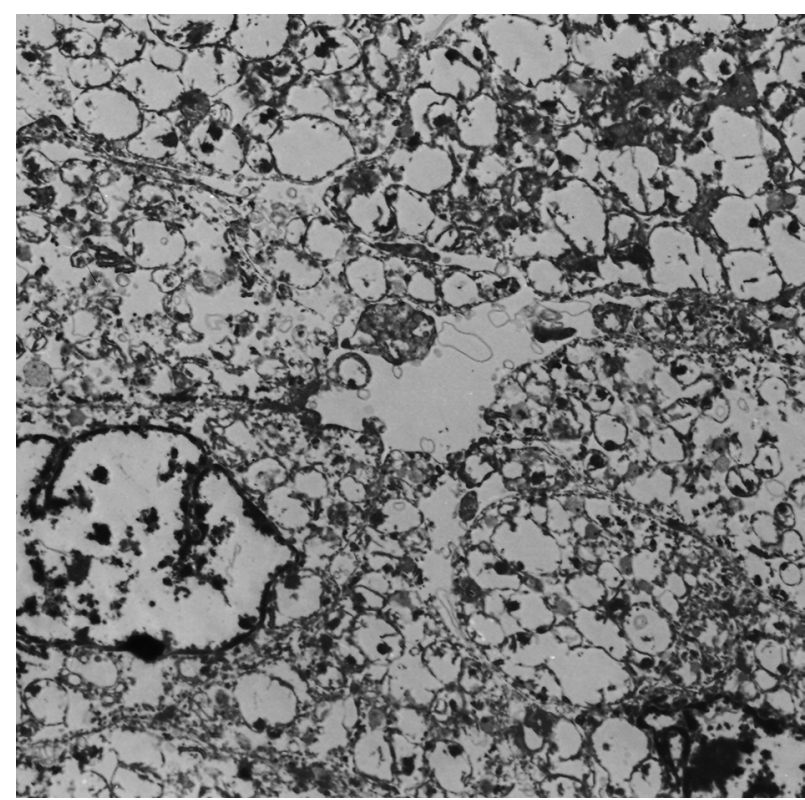

Fig. 3. Electron microscopy reveals oval-shaped tumor cells with abundant mitochondria and shelf-like cristae $(\times 3,000)$.

tubular structures with intraluminal papillary projections have been rarely described. ${ }^{3,4}$ Recent studies reported that a variant of oncocytic RCC having papillotubular growth shows the histology of an extensive small tubular growth pattern, often with papillary fronds. Such tumors are mainly composed of oncocytic cells with eosinophilic granular cytoplasm and some scattered clear vacuolar cells. ${ }^{2-4}$ Either the oncocytic cells or the clear cells in those studies were positive for AMACR, CD10, CK7, epithelial membrane antigen, and vimentin, but they were negative for c-kit and E-cadherin. These histological and immunohistochemical findings are partly shared by those of the present case. Yet in the present case, the microscopic presence of mature fat among the monotonous polygonal cells with oncocytic cytoplasm suggested a possible diagnosis of oncocytoma-like epithelioid AML. Epithelioid AML was often misdiagnosed as granular, chromophobe, or high-grade unclassified RCC until it was included in the classification system of renal tumors. Intratumoral fat may be rarely found in RCC. ${ }^{8}$ Therefore, this uncommonly encountered histologic feature should be distinguished from perinephric and sinus fat invasion of RCC. Negative reaction for melanoma markers, such as HMB-45, Melan-A, microphthalmia transcription factor, and tyrosinase, and immunopositivity for such RCC markers as CD10 and epithelial membrane antigen, can exclude oncocytoma-like epithelioid AML. ${ }^{1}$ The present immunohistochemical results matched those of the tumor cells of papillary RCC and partly matched those of the oncocytic cells of papillary RCC showing papillotubular growth, as re- ported by Masuzawa et al., ${ }^{3}$ whereas oncocytic cells are diffusely stained with AMACR and $\mathrm{CK} 7 .{ }^{6}$ Recent molecular studies that have focused on oncocytic papillary RCC have revealed similar results to those of traditional papillary RCC showing frequent trisomies 7, 12, 16, 17, and 20. ${ }^{1}$ Kunju et al. .5 reported trisomy 7 and 17 and loss of the $Y$ chromosome. Park et al. ${ }^{6}$ demonstrated that comparative genomic hybridization showed gains of $3 \mathrm{p} 22$ and 11q12-q13 in addition to chromosome 17 or loss of 4q. They also demonstrated a loss of chromosome 4 in one case of oncocytic papillary RCC. These molecular changes of oncocytic papillary RCC are similar to other types of papillary RCC. Immunopositivity for AMACR is similar to types 1 and 2 papillary RCC.

The Mallory body-like eosinophilic hyaline globules are not commonly encountered in RCC, but they were first reported by Datta in $1977 . .^{9}$ These structures are morphologically similar to those seen in alcoholic liver disease, which is composed of a parallel array or granular aggregates of intermediate filaments. These prekeratin-like structures show similar immunohistochemical and special staining results as the present case.

The present tumor arose in the background of tuberculous kidney. To date, less than 50 cases of RCC with renal tuberculosis have been reported. ${ }^{10}$ Establishing a causative relationship between the chronic inflammation of tuberculosis and RCC is rather complicated, especially since we know from previous literature that bacillus Calmette-Guérin interaction with the uroepithelium upregulates interleukins and various forms of interferon, which are currently the primary immunotherapies for RCC.

We emphasize a rare morphology of oncocytic RCC with tubulopapillary growth pattern containing a mature fat component. This combination of pathologic findings are rarely encountered and should be distinguished from other types of RCC as well as oncocytoma-like epithelioid AML.

\section{Conflicts of Interest}

No potential conflict of interest relevant to this article was reported.

\section{REFERENCES}

1. Eble JN, Sauter G, Epstein JI, Sesterhenn IA. World Health Organization classification of tumours: pathology and genetics of tumours of the urinary system and male genital organs. Lyon: IARC Press, 2004.

2. Shuch B, Amin A, Armstrong AJ, et al. Understanding pathologic variants of renal cell carcinoma: distilling therapeutic opportunities 
from biologic complexity. Eur Urol 2015; 67: 85-97.

3. Masuzawa N, Kishimoto M, Nishimura A, Shichiri Y, Yanagisawa A. Oncocytic renal cell carcinoma having papillotubular growth: rare morphological variant of papillary renal cell carcinoma. Pathol Int 2008; 58: 300-5.

4. Mai KT, Kohler DM, Robertson SJ, Belanger EC, Marginean EC. Oncocytic papillary renal cell carcinoma with solid architecture: mimic of renal oncocytoma. Pathol Int 2008; 58: 164-8.

5. Kunju LP, Wojno K, Wolf JS Jr, Cheng L, Shah RB. Papillary renal cell carcinoma with oncocytic cells and nonoverlapping low grade nuclei: expanding the morphologic spectrum with emphasis on clinicopathologic, immunohistochemical and molecular features. Hum Pathol 2008; 39: 96-101.

6. Park BH, Ro JY, Park WS, et al. Oncocytic papillary renal cell carcinoma with inverted nuclear pattern: distinct subtype with an indo- lent clinical course. Pathol Int 2009; 59: 137-46.

7. Sironi M, Spinelli M. Oncocytic angiomyolipoma of the kidney: a case report. Int J Surg Pathol 2003; 11: 229-34.

8. Aron M, Aydin H, Sercia L, Magi-Galluzzi C, Zhou M. Renal cell carcinomas with intratumoral fat and concomitant angiomyolipoma: potential pitfalls in staging and diagnosis. Am J Clin Pathol 2010; 134: 807-12.

9. Datta BN. Hyaline intracytoplasmic globules in renal carcinoma. Arch Pathol Lab Med 1977; 101: 391.

10. Tickoo SK, dePeralta-Venturina MN, Harik LR, et al. Spectrum of epithelial neoplasms in end-stage renal disease: an experience from 66 tumor-bearing kidneys with emphasis on histologic patterns distinct from those in sporadic adult renal neoplasia. Am J Surg Pathol 2006; 30: 141-53. 